\title{
Utilização dos Centros de Atenção Psicossocial (CAPS) na cidade de Santos, São Paulo, Brasil
}

\author{
Utilization of community mental health services \\ in the city of Santos, São Paulo, Brazil
}

\footnotetext{
1 Programa de Saúde

Coletiva, Universidade

Católica de Santos,

Santos, Brasil.

2 Núcleo de Metodologia

e Estatística Aplicadas,

Departamento de

Psiquiatria, Universidade

Federal de São Paulo,

São Paulo, Brasil.

3 Secretaria Municipal

de Saúde de Santos,

Santos, Brasil.

4 Departamento de

Psicologia, Universidade

Católica de Santos,

Santos, Brasil.

5 Setor de Psiquiatria

Social, Departamento de

Psiquiatria, Universidade

Federal de São Paulo,

São Paulo, Brasil.

Correspondência Sérgio Baxter Andreoli Rua Dr. Carvalho de

Mendonça 144, Santos, SP 11070-906, Brasil.

andreoli@psiquiatria.epm.br
}

\begin{abstract}
To report on the clientele treated at community mental health services (CAPS) in the city of Santos, São Paulo, Brazil, a census was conducted of the patients examined at the services from June 1, 2001, to June 30, 2001. The patient's initial contact with the service was registered, and data were recorded on a standardized form, using the medical case history as a source of information. Among the 1,673 patients, average age was 46 years, 59.0\% were female, 58.0\% lived with their families, and mean duration of treatment was three years. Some 38.0\% were schizophrenic, schizotypical, or in acute delusional states, $31.0 \%$ presented mood disorders, and $17.0 \%$ were neurotic. Treatment modalities included out-patient psychiatric (81.0\%), individual (23.0\%), group (13.0\%), and psychosocial rehabilitation (4.3\%). In Santos, CAPS treat all categories of mental disorders (and frequently those considered the most severe cases) expected at this type of service. The treatment model is that of an out-patient psychiatric clinic.
\end{abstract}

Mental Disorders; Health Service; Mental Health; Health Policy
Sérgio Baxter Andreoli 1,2

Simone de Souza B. Ronchetti ${ }^{3}$

Ana Lúcia Pimenta de Miranda ${ }^{3}$

Claudia Rodrigues Monteiro Bezerra ${ }^{3}$

Catulo César Pestana de Barros Magalhães 4

Denise Martin 1,5

Rosa Maria Ferreiro Pinto 1

\section{Introdução}

A reforma da atenção psiquiátrica na cidade de Santos, São Paulo, tem sido considerada como um exemplo para a elaboração de estratégias e modelos de políticas em saúde mental no Brasil. Quando da sua implantação, em maio de 1989, teve repercussão internacional, principalmente por colocar em prática a política de desospitalização do paciente psiquiátrico de modo agudo, por meio da desativação do hospital psiquiátrico da cidade. Desativado o hospital, a organização dos serviços foi sendo estruturada ao longo dos anos em Centros Comunitários de Atenção Psicossocial (CAPS).

\section{Organização da rede \\ de assistência municipal}

A rede municipal de atenção à saúde mental na cidade de Santos atendeu aproximadamente 8.000 pacientes no ano de 2001, segundo dados da Coordenadoria de Saúde Mental do Município, e está composta dos seguintes equipamentos: cinco Seções Núcleo de Atenção Psicossocial (CAPS); uma Seção Reabilitação Profissional (SeRP); uma Seção Núcleo de Atenção ao Tóxico-Dependente (SeNAT); uma emergência psiquiátrica e uma Seção Lar Abrigado (SeLAb). Os CAPS são responsáveis pela maior parte do atendimento prestado ao paciente psiquiátrico da cidade, o qual é regionalizado e funciona 24 
horas. Antes de entrar no sistema, o paciente passa por uma triagem feita por um profissional ligado ao serviço. Uma vez aceito, passará por uma avaliação psiquiátrica. Caso tenha possibilidade de ser reabilitado profissionalmente é encaminhado para a SeRP.

As modalidades de atendimento nos CAPS são: (1) hospitalidade integral, na qual o usuário permanece internado; (2) hospitalidade diurna; (3) hospitalidade noturna; (4) atendimentos individuais, realizados exclusivamente com o usuário e família na forma de entrevistas, aconselhamento ou intervenções terapêuticas de diversas naturezas; (5) atendimentos grupais, realizados pelos profissionais da unidade na forma de assembléias, grupos operativos, grupos terapêuticos e outras atividades das quais pode participar a família; (6) ambulatório médico-psiquiátrico de acompanhamento clínico; (7) abordagens extra-institucionais realizadas com ou para o usuário e família, tais como visitas domiciliares, passeios, acompanhamento e interconsultas; (8) reabilitação psicossocial para os usuários que integram alguma ação de caráter específico de reabilitação, tais como os projetos da SeRP ou centro de convivência e (9) outros, tais como triagem e medicação assistida. Esta última consiste na administração ou fornecimento de medicação garantindo a continuidade do tratamento.

\section{Organização da rede}

de assistência estadual

O município conta com mais dois equipamentos, uma enfermaria psiquiátrica no hospital geral (Hospital Guilherme Álvaro) e um ambulatório, cuja gestão é do governo estadual e o modelo assistencial é médico-psiquiátrico. Ambos atendem pacientes provenientes da Baixada Santista, São Paulo, e não apenas pacientes da cidade. A cidade conta ainda com leitos em hospital psiquiátrico de referência na cidade de São João da Boa Vista, São Paulo, distante aproximadamente $200 \mathrm{~km}$.

\section{Centros de Atenção Psicossocial}

Os CAPS foram redefinidos recentemente, no âmbito federal, pela Portaria no 336 (Diário Oficial da União; 2002, 20 Fev) a qual os estabelece em três níveis: CAPS 1, CAPS 2 e CAPS 3 , definidos por ordem crescente de porte, complexidade e abrangência populacional. As três modalidades de serviços cumprem a mesma função no atendimento público em saúde mental, devendo se constituir em serviço ambulatorial de atenção diária. Deverão estar capacitados para realizar prioritariamente o atendimento de pacientes com transtornos mentais graves e persistentes em sua área territorial, em regime de tratamento intensivo, semi-intensivo e não-intensivo.

O destaque dado aos CAPS na atual política de saúde mental pode ser avaliado dentro de um quadro comparativo relacionando o número destes serviços em 2001 e 2002. No Brasil, em 2001, eram 295 (0,017CAPS/ 10 mil habitantes) e passaram a $423(0,024 \mathrm{CAPS} / 10 \mathrm{mil}$ habitantes) em 2002. A distribuição desse tipo de equipamento pelo território nacional é desigual. O Rio Grande do Sul encabeça a lista (0,051CAPS/ 10 mil habitantes) e os estados da Região Norte não possuem nenhum. O Estado de São Paulo ocupa a 12a posição e passou de 48 (0,013CAPS/10 mil habitantes) para 105 $(0,027 \mathrm{CAPS} / 10 \mathrm{mil}$ habitantes$)$ no referido período (dados disponíveis no endereço eletrônico do DATASUS: http://www.datasus.gov.br/ tabnet/tabnet.htm; acessado em 28/Ago/2003).

A rede de assistência à saúde mental na cidade de Santos é baseada em CAPS. No total são cinco (0,12CAPS/10 mil habitantes), estão bem distribuídos pela cidade e possuem uma estrutura estável, há pelo menos dez anos. Este número está um pouco acima daquele considerado como o suficiente para uma cidade desse porte (418 mil habitantes). Thornicroft \& Tansella 1 preconizam um serviço comunitário para uma área de captação de 150 mil a 200 mil habitantes.

Dadas essas características e a importância dessa rede no cenário nacional, nosso objetivo foi descrever a clientela atendida nos serviços de atenção comunitária de saúde mental da cidade de Santos nos aspectos demográficos, nos tipos de modalidades de atendimentos oferecidos e nas características clínicas, com o propósito de fornecer subsídios para sua avaliação e alguns parâmetros para o desenvolvimento de políticas públicas em saúde mental.

\section{Método}

Foi realizado um censo dos pacientes atendidos nos CAPS da rede de assistência municipal de Santos durante o mês de junho de 2001 (01 de junho de 2001 a 30 de junho de 2001), incluindo fins de semana e feriados. Na ocasião, foram recolhidos dados demográficos sobre a 
modalidade de atendimento prestado e dados clínicos de cada um dos pacientes.

\section{Procedimentos de coleta de dados}

Para cada local foi designado um pesquisador responsável e aberto um livro no qual eram registrados o número do prontuário médico, nome e data, isso para cada usuário que comparecesse ao serviço durante o mês de coleta dos dados. A lista de usuários do dia, obtida no balcão de atendimento, era confrontada com a lista do livro do pesquisador com o objetivo de excluir os retornos. Os dados do primeiro registro do usuário na unidade naquele mês permitiram sua identificação e, com base nesses, foram preenchidas as fichas padronizadas com informações colhidas diretamente dos prontuários. Quando não havia informação suficiente, os pesquisadores tentavam recuperálas diretamente com os profissionais envolvidos no tratamento.

\section{Análise dos dados}

A análise descritiva dos dados seguiu três eixos: demográfico, modalidade de atendimento e clínico. Para cada um, foram examinadas as tabelas de contingência desagregada por sexo, pelos diferentes serviços e pelos três grupos diagnósticos mais freqüentes (transtornos esquizofrênicos, humor e neuróticos). As comparações foram feitas por meio das proporções observadas.

\section{Resultados}

A qualidade dos dados pôde ser considerada boa, quando avaliada pelas perdas de informações nas diferentes variáveis. No geral, as variáveis demográficas apresentaram porcentagens variando entre $1,0 \%$ e $3,0 \%$. No grupo das modalidades de atendimento, as porcentagens de perdas foram menores do que $0,2 \%(n=3)$. No grupo das clínicas, as perdas variaram entre $1,6 \%$ e $16,0 \%$, com a menor taxa registrada para o diagnóstico psiquiátrico e a maior para o número de internações ao longo da vida. A exceção foi verificada na variável naturalidade, a qual apresentou perda de 45,0\% $(n=782)$.

Nos CAPS foram atendidos 1.673 usuários (Tabela 1), 60,0\% do sexo feminino, com idade média de 46 anos (variação: 1-86; dp = 14), $67,0 \%$ natural de cidades da região metropolitana da Baixada Santista (Santos, Cubatão, Iguape, São Vicente), 52,0\% natural de Santos, 78,0\% morando com a família $(34,0 \%$ originária e
$45,0 \%$ conjugal) e $98,0 \%$ procedente da zona de captação do serviço em que se consultou.

As modalidades de atendimentos mais utilizadas nos últimos trinta dias foram o ambulatório médico-psiquiátrico $(82,0 \%)$ e outras modalidades $(37,0 \%)$ (Tabela 1$)$, dentre as quais 93,0\% foram medicação assistida. No perfil clínico, podemos observar três grupos diagnósticos psiquiátricos principais, por ordem de freqüência: transtornos psicóticos (esquizofrênicos, esquizotípicos e delirantes) corresponderam a $38,0 \%$ dos atendimentos; de humor a $32,0 \%$ e neuróticos a $17,0 \%$ (Tabela 1).

\section{Esquizofrenias}

No grupo das esquizofrenias foram atendidos 629 pacientes com os seguintes diagnósticos específicos: esquizofrênicos $(49,8 \%)$, psicose não-orgânica e não-especificada $(37,5 \%)$, esquizotípico $(0,3 \%)$, delirante persistente $(2,1 \%)$, psicose aguda transitória $(5,3 \%)$, delirante induzido $(0,2 \%)$, esquizoafetivo $(3,6 \%)$ e outros transtornos psicóticos não-orgânicos (1,6\%). Na descrição por gêneros, observamos $53,0 \%$ de homens, com 42 anos de média de idade (variação: $17-79$; dp = 12) e 59,0\% vivendo com a família originária. As mulheres apresentaram 46 anos de média de idade (variação: 8-86; $\mathrm{dp}=$ $14), 38,0 \%$ vivendo com a família originária e $38,0 \%$ com a família conjugal.

O grupo das esquizofrenias utilizou todos os tipos de atendimentos do serviço, numa proporção maior do que os pacientes com outros diagnósticos, sobretudo os de hospitalidade, atendimento individual, grupal, reabilitação e medicação assistida (Tabela 2). Esta utilização entretanto não foi diferente entre os gêneros.

Em relação às características clínicas (Tabela 2), iniciaram a sintomatologia em média aos 27 anos (mediana = 24; variação: $2-74$; $\mathrm{dp}=$ 11), os homens aos 25 (variação: $4-73$; dp = 11) e as mulheres aos 28 anos (variação:2-74; dp = 12). Apresentaram média de 4 anos de tratamento nos serviços (mediana $=4$; variação: 0 10 ; $\mathrm{dp}=3$ ); média de três internações na vida (mediana $=1$; variação: $0-55 ; \mathrm{dp}=5 ; 37,0 \%$ com três ou mais internações) e 74,0\% tiveram tratamento anterior. Apenas com relação ao número de internações houve diferença entre os gêneros, pois $28,0 \%$ das mulheres tiveram três ou mais contra $38,0 \%$ dos homens. Em relação à associação com diagnóstico clínico mostraram uma freqüência de $9,0 \%$, a qual foi menor do que aquelas observadas nos outros diagnósticos. 
Caracterização dos pacientes atendidos em cada um dos cinco Centros de Atenção Psicossocial de Santos, São Paulo, Brasil ( $n=1.673$ atendidos em junho de 2001).

\begin{tabular}{|c|c|c|c|c|c|c|}
\hline & 1 & 2 & $\begin{array}{c}\text { CAPS } \\
3\end{array}$ & 4 & 5 & Total \\
\hline Homens (\%) & 39,1 & 42,1 & 40,6 & 44,2 & 37,1 & 40,2 \\
\hline Idade média em anos (variação, $d p)(n=1.610)$ & $47(14-84 ; 15)$ & $42(11-75 ; 13)$ & $46(1-86 ; 15)$ & $46(18-87 ; 14)$ & $47(18-85 ; 15)$ & $46(1-87 ; 15)$ \\
\hline Natural de Santos $(\%)(n=926)$ & 49,9 & 45,1 & 69,6 & 41,8 & 61,7 & 52,4 \\
\hline Vive na zona de captação (\%) $(n=1.655)$ & 97,1 & 97,9 & 99,4 & 99,7 & 99,7 & 98,6 \\
\hline \multicolumn{7}{|l|}{ Com quem vive $(n=1.617)$} \\
\hline Sozinho (\%) & 8,3 & 16,2 & 6,4 & 11,2 & 9,0 & 9,3 \\
\hline Família originária (\%) & 32,7 & 31,7 & 30,5 & 35,1 & 38,0 & 33,7 \\
\hline Família conjugal (\%) & 47,2 & 35,2 & 47,3 & 38,4 & 47,2 & 44,7 \\
\hline Parentes/amigos (\%) & 10,2 & 10,6 & 14,5 & 12,7 & 3,5 & 10,0 \\
\hline Sem residência fixa (\%) & 0,6 & 2,8 & 0,3 & 1,5 & 1,2 & 1,0 \\
\hline \multicolumn{7}{|l|}{ Tipo de atendimento nos últimos trinta dias $(n=1670)$} \\
\hline Hospitalidade integral (\%) & 2,9 & 6,3 & 1,0 & 2,3 & 3,1 & 2,8 \\
\hline Hospitalidade diurna (\%) & 3,2 & 11,8 & 2,9 & 4,9 & 6,9 & 5,0 \\
\hline Hospitalidade noturna (\%) & 0,9 & 0,7 & 2,6 & 0,7 & 1,1 & 1,2 \\
\hline Atendimentos individuais (\%) & 33,2 & 46,5 & 1,3 & 10,7 & 28,9 & 23,4 \\
\hline Atendimentos grupais (\%) & 26,4 & 8,3 & 2,6 & 3,9 & 12,3 & 13,3 \\
\hline Ambulatório médico-psiquiátrico (\%) & 69,5 & 83,3 & 90,4 & 85,7 & 93,1 & 82,5 \\
\hline Abordagens extra-institucionais (\%) & 1,1 & 5,6 & 1,0 & 2,3 & 2,9 & 2,0 \\
\hline Reabilitação psicossocial (\%) & 5,9 & 7,6 & 1,3 & 5,2 & 2,0 & 4,3 \\
\hline Outro (\%) & 94,4 & 3,4 & 1,6 & 20,8 & 5,4 & 37,0 \\
\hline $\begin{array}{l}\text { Número médio de anos em tratamento } \\
\text { no serviço (variação; } d p)(n=1.608)\end{array}$ & $3,6(0-10 ; 3)$ & $4,6(0-10 ; 3)$ & $2,8(0-10 ; 3)$ & $3,6(0-10 ; 3)$ & $2,9(0-10 ; 3)$ & $3,4(0-10 ; 3)$ \\
\hline Internações na vida (mediana) (variação) ( $n=1.402)$ & $0(0-40)$ & $1(0-30)$ & $0(0-49)$ & $0(0-55)$ & $1(0-26)$ & $0(0-55)$ \\
\hline Tem tratamento anterior $(\%)(n=1.616)$ & 68,9 & 65,7 & 49,7 & 69,8 & 60,3 & 63,3 \\
\hline Tem diagnóstico clínico associado $(\%)(n=1.673)$ & 13,3 & 13,1 & 6,7 & 10,7 & 35,4 & 16,2 \\
\hline \multicolumn{7}{|l|}{ Grupo diagnóstico pelo CID-10 ( $n=1.673)$} \\
\hline Transtorno de humor (\%) & 28,9 & 30,9 & 38,4 & 30,7 & 31,3 & 31,7 \\
\hline Esquizofrenia, esquizotípico e delirantes (\%) & 43,6 & 44,9 & 36,1 & 43,5 & 23,8 & 38,1 \\
\hline Transtornos neuróticos (\%) & 14,6 & 11,0 & 16,5 & 16,0 & 26,7 & 17,5 \\
\hline Retardo mental (\%) & 5,2 & 8,8 & 1,6 & 2,0 & 8,4 & 4,9 \\
\hline Transtornos orgânicos (\%) & 4,7 & 2,2 & 3,2 & 3,6 & 6,7 & 4,4 \\
\hline Álcool e substâncias (\%) & 2,4 & 1,5 & 2,3 & 2,3 & 2,9 & 2,4 \\
\hline Outros (\%) & 0,5 & 0,7 & 1,9 & 2,0 & 0,3 & 1,0 \\
\hline Número total de pacientes & 557 & 145 & 313 & 308 & 350 & 1673 \\
\hline
\end{tabular}

CAPS = Centros de Atenção Psicossocial. Os vários tipos de atendimentos não são excludentes. 
Caracterização dos pacientes atendidos nos Centros de Atenção Psicossocial de Santos, São Paulo, Brasil, pelos grupos diagnósticos CID-10 (esquizofrenia, transtornos esquizotípicos e delirante, transtornos do humor e transtornos neuróticos) ( $n=1.673$ atendidos em junho de 2001).

\begin{tabular}{|c|c|c|c|}
\hline & Esquizofrenias & Transtornos de humor & Neuróticos \\
\hline Homens (\%) & 52,9 & 25,0 & 27,4 \\
\hline Idade Média (mediana; max-min; dp) & $44(43 ; 8-86 ; 13)$ & $49(48 ; 1-87 ; 15)$ & $48(46 ; 9-85 ; 15)$ \\
\hline Natural de Santos (\%) & 56,2 & 47,1 & 50,6 \\
\hline \multicolumn{4}{|l|}{ Com quem vive } \\
\hline Sozinho (\%) & 10,1 & 8,6 & 9,4 \\
\hline Família originária (\%) & 49,3 & 14,7 & 21,7 \\
\hline Família conjugal (\%) & 28,2 & 62,2 & 60,6 \\
\hline Parentes ou amigos (\%) & 10,5 & 12,0 & 5,4 \\
\hline Instituto, casa de repouso ou lar abrigado (\%) & 0,5 & 2,2 & 1,5 \\
\hline Sem residência & 0,5 & 0,0 & 0,0 \\
\hline \multicolumn{4}{|l|}{ Tipo de atendimento nos últimos trinta dias } \\
\hline Hospitalidade integral (\%) & 4,0 & 2,9 & 0,3 \\
\hline Hospitalidade diurna (\%) & 8,6 & 3,3 & 1,0 \\
\hline Hospitalidade noturna (\%) & 1,6 & 1,1 & 0,7 \\
\hline Atendimento individual (\%) & 27,0 & 23,3 & 18,5 \\
\hline Atendimento grupal (\%) & 13,1 & 11,7 & 17,8 \\
\hline Ambulatório médico-psiquiátrico (\%) & 81,7 & 84,5 & 81,5 \\
\hline Abordagens extra-institucionais (\%) & 1,4 & 1,5 & 2,4 \\
\hline Reabilitação psicossocial (\%) & 7,3 & 3,1 & 0,7 \\
\hline Medicação assistida (\%) & 42,6 & 31,1 & 27,1 \\
\hline \multicolumn{4}{|l|}{ Características clínicas } \\
\hline $\begin{array}{l}\text { Idade de início dos sintomas, média } \\
\text { (mediana; max-min; dp) }\end{array}$ & $26,8(24 ; 2-74 ; 11)$ & $36,4(35 ; 2-84 ; 16)$ & $36,5(35 ; 5-80 ; 14)$ \\
\hline $\begin{array}{l}\text { Número médio de anos de tratamento } \\
\text { no serviço (mediana; max-min; } d p)\end{array}$ & $4,4(4 ; 0-10 ; 3)$ & $2,6(1 ; 0-10 ; 3)$ & $2,4(1 ; 0-9 ; 3)$ \\
\hline $\begin{array}{l}\text { Número médio de internações na vida } \\
\text { (mediana; max-min; dp) }\end{array}$ & $3(1 ; 0-55 ; 5)$ & $1,4(0 ; 0-26 ; 3)$ & $0,3(0 ; 0-9 ; 1)$ \\
\hline Tem tratamento anterior (\%) & 74,1 & 56,2 & 50,2 \\
\hline Tem diagnóstico clínico associado (\%) & 8,6 & 17,6 & 25,7 \\
\hline Número total de pacientes $(n)$ & 629 & 523 & 288 \\
\hline
\end{tabular}

CAPS $=$ Centros de Atenção Psicossocial. Obs: os vários tipos de atendimentos não são excludentes.

\section{Esquizofrenias de primeiro contato}

Os pacientes do grupo diagnóstico das esquizofrenias de primeiro contato são importantes clinicamente e no contexto da saúde pública, sobretudo pelo curso crônico desses transtornos. Neste estudo foram definidos entre aqueles com menos de um ano em tratamento no serviço e sem tratamento anterior. No total foram 36 usuários, correspondendo a 6,0\% do total das esquizofrenias, e com as seguintes características: $61,0 \%$ de homens, média de ida- de de 35 anos (mediana = 34; variação: 14-86; $d p=15)$ e $37,0 \%$ moram com a família originária. Os diagnósticos específicos ficaram assim distribuídos: $22,0 \%$ de esquizofrênicos, $61,0 \%$ de psicose não-orgânica e não-especificada, $6,0 \%$ de outros transtornos psicóticos e 11,0\% de psicóticos agudos transitórios. Os dois serviços que mais receberam pacientes de primeiro contato foram o CAPS $1(n=13)$ e o CAPS 3 $(\mathrm{n}=14)$. 


\section{Transtornos do humor}

No grupo dos transtornos de humor foram atendidos 523 pacientes, sendo 2,0\% com episódio maníaco, $18,0 \%$ transtorno afetivo bipolar, $59,0 \%$ episódio depressivo, $7,0 \%$ transtorno depressivo recorrente, $6,0 \%$ transtorno de humor (afetivo) persistente, $0,4 \%$ outros transtornos de humor e $6,0 \%$ transtorno de humor (afetivo) não-especificado. Setenta e cinco porcento eram mulheres, a média de idade foi de 49 anos (mediana $=48$; variação: $1-87$; $d p=15)$ e $62,0 \%$ viviam com a família conjugal (Tabela 2 ). Na comparação entre gêneros, observou-se uma tendência das mulheres morarem com a família conjugal, foram $66,0 \%$ contra $50,0 \%$ dos homens.

Os principais tipos de atendimentos utilizados por esses pacientes foram (Tabela 2): ambulatório médico-psiquiátrico (84,0\%); medicação assistida $(31,0 \%)$ e atendimento grupal $(12,0 \%)$. Não foram observadas diferenças entre os gêneros. Em relação aos outros dois diagnósticos estudados, não utilizaram as modalidades de atendimento tanto quanto o grupo das esquizofrenias, mas, em relação ao grupo dos neuróticos, apresentaram maior utilização, sobretudo nas modalidades de hospitalização, atendimento individual e reabilitação.

Em relação às características clínicas, a média de idade de início da sintomatologia foi de 36 anos (mediana = 35; variação: $2-84 ; \mathrm{dp}=16$ ), a média do tempo de tratamento no serviço foi de três anos (mediana = 1; variação: $0-10 ; \mathrm{dp}=$ 3), a do número de internação na vida foi de um (mediana = 0; variação: $0-26 ; d p=3 ; 19,0 \%$ com três ou mais internações), 56,0\% tiveram tratamento anterior e $18,0 \%$ apresentaram associação com diagnóstico clínico. Nenhuma destas variáveis apresentou diferenças entre os gêneros.

\section{Transtornos neuróticos}

No grupo com transtornos neuróticos foram atendidos 288 pacientes, sendo $8,5 \%$ com transtornos fóbico-ansiosos, $63,0 \%$ outros transtornos ansiosos, 5,0\% transtornos obsessivo-compulsivos, 10,0\% reação ao estresse grave e transtorno de adaptação, 7,0\% transtorno dissociativo (de conversão), 2,0\% transtorno somatoforme e $25,0 \%$ outros transtornos neuróticos. Setenta e três por cento eram mulheres, com idade média de 48 anos (mediana $=46$; variação: 9-85; dp = 15) e 61,0\% morando com a família conjugal. A única diferença entre gêneros foi observada entre as médias de idade, as mulheres apresentaram 49 anos (mediana $=49$; variação: 20-85; dp = 15) e os homens 44 (mediana $=44$; variação: 9-83; $\mathrm{dp}=15$; um paciente com menos de 18 anos).

A idade média de início dos sintomas foi de 36 anos (mediana = 35; variação: $5-80 ; d p=14$ ), o tempo médio em tratamento no serviço foi de três anos (mediana = 1; variação: $0-10 ; \mathrm{dp}=$ 3 ), $88,0 \%$ nunca foram internados, $27,0 \%$ têm diagnóstico clínico associado e 50,0\% teve tratamento anterior. Nenhuma diferença foi encontrada entre os gêneros.

A utilização das modalidades de atendimento concentrou-se nos serviços de ambulatório médico-psiquiátrico $(81,0 \%)$, medicação assistida $(27,0 \%)$, atendimento individual $(18,0 \%) \mathrm{e}$ atendimento grupal $(18,0 \%)$. Os demais atendimentos ficaram com apenas uma parcela de $5,0 \%$ de usuários.

\section{Diferenças entre os CAPS}

O número de pacientes atendidos foi diferente entre os CAPS durante o mês de coleta dos dados. Quando comparamos os serviços, observamos que o CAPS 2 atendeu $8,0 \%$ do total de usuários; o CAPS 1, 32,0\% e os outros ficaram com proporções variando em torno dos $18,0 \%$.

O perfil do tipo de atendimento oferecido aos usuários em cada CAPS também variou e pode ser verificado na Tabela 1 . O CAPS 1 , apresentou $33,0 \%$ de atendimento individual, $26,0 \%$ grupal e $94,0 \%$ de outros atendimentos $(93,0 \%$ de medicação assistida) e 69,0\% para o atendimento médico-psiquiátrico. Na comparação entre os serviços, portanto, observamos que o CAPS 1 apresentou proporções maiores de atendimento nas primeiras modalidades e menores na última (Tabela 1). Parte desta característica pode ser explicada pela forma de atendimento utilizada na modalidade médico-psiquiátrica, pois, apenas no CAPS 1 ela é feita em grupo.

Examinando ainda a Tabela 1, comparativamente em relação aos tipos de modalidades de atendimento, observamos algumas diferenças entre os serviços. O CAPS 2 apresentou $46,0 \%$ de atendimento individual, $12,0 \%$ hospitalidade diurna e $0,7 \%$ nos outros atendimentos. O CAPS 3 caracterizou-se por apresentar 90,0\% de atendimento médico-psiquiátrico e nas demais modalidades proporções menores do que 3,0\%. O CAPS 4, 21,0\% de medicação assistida. O CAPS 5, 93,0\% nos atendimentos médico-psiquiátrico, 29,0\% individuais e 7,0\% hospitalidade diurna.

O perfil clínico dos usuários em cada CAPS também variou muito e pode ser verificado na Tabela 1. Em relação aos diagnósticos, os CAPS 3 e 4 se destacaram pela proporção de $90,0 \%$ de 
usuários com transtornos de humor, esquizofrênicos e neuróticos e, de outro lado, o CAPS 5 com uma distribuição mais variada de outros diagnósticos. Examinando a distribuição dos três grupos diagnósticos mais freqüentes, observamos que os CAPS 1,2 e 4 atendem em torno de $44,0 \%$ de esquizofrênicos e $30,0 \%$ de transtorno de humor. No CAPS 3 , a diferença entre as proporções de esquizofrênicos $(36,0 \%)$ e as de transtorno de humor $(38,0 \%)$ é menor e inverte a ordem do diagnóstico mais prevalente. No CAPS 5, diminuem as diferenças entre as proporções dos três diagnósticos, aumentando a de transtorno neurótico $(27,0 \%)$.

Em relação ao número de anos em tratamento nos serviços, as médias variaram entre 2,8 a 4,6 anos, com os CAPS 1, 2 e 4 apresentando as maiores médias. Os CAPS 3 e 5 apresentaram as menores proporções em relação a ter tratamento anterior, respectivamente $50,0 \%$ e $60,0 \%$ (coincide com a proporção alta de atendimento médico-psiquiátrico). Ter diagnóstico clínico associado foi uma característica do paciente que marcou duas diferenças importantes entre os CAPS. Por um lado, o CAPS 5 com a proporção de $35,0 \%$ e, por outro, o CAPS $3 \operatorname{com} 7,0 \%$.

\section{Discussão}

Embora a reforma psiquiátrica na cidade de Santos tenha sido objeto de muitas discussões ao longo dos últimos anos, sobretudo daquelas que culminaram na Lei 10.216 (Diário Oficial da União 2001; 4 Set) que trata da proteção e direitos das pessoas portadoras de transtornos mentais, a maior parte dos trabalhos científicos originados com base nessa experiência trata das concepções teóricas do modelo de assistência proposto. Existem poucos estudos empíricos sobre as modalidades de atendimento e perfil de pacientes publicados em periódicos, 2,3 e a maioria foi descrita preferencialmente na forma de relatos de casos publicados em teses ou relatórios de pesquisa.

Nosso estudo, embora ainda limitado por se tratar de um censo de um mês e ter incluído apenas os CAPS, procurou em parte preencher esta lacuna. Os centros comunitários de assistência à saúde mental estudados realizam todos os tipos de modalidades de atendimento; da distribuição de medicamentos à internação psiquiátrica. Mas são caracterizados sobretudo pelo atendimento médico-psiquiátrico, ou seja, com características de funcionamento ambulatorial. Todos os tipos de transtornos psiquiátricos foram encontrados e a proporção de atendimentos seguiu o que é considerado padrão de necessidade da população para a promoção da saúde mental 4 . Ou seja, os transtornos mais prevalentes são atendidos em maior proporção, tais como o de humor e os neuróticos; assim como os transtornos mais graves e crônicos, embora menos prevalentes, tais como os esquizofrênicos.

Outros resultados apontam também na direção de uma demanda compatível com a que é esperada em centros comunitários. $\mathrm{O}$ perfil clínico de paciente crônico é um deles 5 e pôde ser mostrado por meio das médias altas de idade, prevalência alta de indivíduos com tratamento anterior, média alta do número de internações na vida e média alta de anos em tratamento no serviço. O perfil clínico do grupo de pacientes esquizofrênicos está de acordo com o que tem sido encontrado na literatura, sobretudo aquele relativo à idade de início dos sintomas, qual seja, no final da adolescência e diferente entre os gêneros; homens com início mais precoce 6 .

A demanda examinada entre os CAPS mostrou diferenças grandes e, ao nosso ver, existiu uma adequação entre o perfil de morbidade mais prevalente e a distribuição dos cuidados oferecidos. Assim, no CAPS 3 observou-se maior proporção de atendimentos aos pacientes com transtorno de humor e maior proporção no atendimento médico-psiquiátrico. No CAPS 5, observou-se maior proporção de transtornos neuróticos e outros transtornos com maior proporção de atendimento médico-psiquiátrico, mas também de atendimentos grupais e individuais. O CAPS 2 apresentou uma proporção de pacientes atendidos bem menor $(8,0 \%)$, mas atendeu pacientes mais graves, tais como os esquizofrênicos, com idade de início mais precoce, tempo maior de tratamento no serviço e com maior número de internações na vida. Estas características resultaram numa oferta de atendimentos mais variada do que aquela observada nos demais serviços, sobretudo naqueles de reabilitação psicossocial e abordagens extra-institucionais.

Dentro da proposta de avaliação da rede de assistência em Santos, segundo o modelo matricial de avaliação proposto por Thorncroft \& Tansella 1, este trabalho pode ser considerado a parte que avalia o processo local. Outras dimensões importantes não foram avaliadas, tais como a disponibilidade de recursos humanos e sua capacitação, ou os resultados relativos à redução de sintomas e qualidade de vida dos pacientes. Um dos poucos indicadores considerados de resultado que pudemos levantar foi o número de internações hospitalares de pacien- 
tes provenientes de Santos. Esse indicador é considerado importante para uma avaliação de CAPS, pois esse tipo de serviço foi criado com o objetivo de oferecer uma alternativa ao hospital psiquiátrico.

O número de internações na rede pública hospitalar de pacientes provenientes de Santos, no ano de 2001, foi de 121 , sendo 80 internados na enfermaria psiquiátrica do Hospital Guilherme Álvaro, sendo os leitos na cidade, e 41 no Hospital Psiquiátrico de São João da Boa Vista, portanto leitos fora da cidade. Sessenta e três porcento com diagnóstico dentro do grupo de psicóticos da CID-10 (esquizofrênicos, esquizotípicos e transtornos delirantes) (F20F29) e $19,0 \%$ transtornos comportamentais e mentais devido ao uso de álcool 7 .

Um cenário hipotético de necessidades de hospitalização na cidade pode ser construído baseando-se em resultados de estudos epidemiológicos. Estes encontraram $0,6 \%$ de demanda potencial de tratamento para o paciente psicótico na população geral 8 , que $30,0 \%$ desses são graves e demandam hospitalizações repetidas 9 . Dentre os hospitalizados, $50,0 \%$ são internados novamente caso não sejam tratados adequadamente na comunidade 10 . Baseado neste cenário, estimamos que 416 pacientes necessitariam de hospitalização na Cidade de Santos, caso não estivessem encontrando tratamento adequado na comunidade. Comparando esse número hipotético com os 121 internados em 2001, destes 76 (63,0\%) psicóticos, podemos concluir que os CAPS de Santos estão cumprindo seus objetivos de desospitalização.

\section{Resumo}

A fim de descrever a clientela atendida nos Centros de Atenção Psicossocial (CAPS) da Cidade de Santos foi realizado um censo de pacientes atendidos nos CAPS no período 1o de junho de 2001 a 30 de junho de 2001. O primeiro contato do paciente com o serviço foi registrado e seus dados foram preenchidos em ficha padronizada utilizando o prontuário médico como fonte de informação. Foram atendidos 1.673 usuários, com idade média de 46 anos, mulheres (59,0\%), morando com a família (78,0\%) e tempo médio de três anos em tratamento no serviço. Atenderam 38,0\% de esquizofrênicos, esquizotípicos e delirantes, 31,0\% com trans-

\section{Limitações}

Alguns cuidados devem ser tomados ao examinarmos nossos resultados. O primeiro deles e o mais importante, é que (1) nosso levantamento registrou apenas o primeiro contato do paciente no mês, o que pode levar a interpretações falsas sobre o volume de atendimentos realizados pelo serviço. No caso dos pacientes graves, o número de atendimentos pode ser diário; (2) a alta proporção de regionalização encontrada, acima de $97,0 \%$, foi considerada superestimada pelas autoridades locais, isso porque, Santos é a cidade de referência na Baixada Santista e referência nacional para tratamento de saúde mental, portanto atrai pacientes de outras cidades e estados, os quais permanecem temporariamente morando no município e (3) a alta prevalência de associação com doença clínica, sobretudo entre os pacientes neuróticos e com transtorno de humor, aponta para a necessidade de uma integração do sistema de saúde, entretanto, nosso estudo não foi capaz de investigar como esta integração tem se dado.

\section{Conclusão}

Os CAPS de Santos atendem todas as categorias de transtornos mentais, sendo os mais freqüentes aqueles considerados mais graves, o que é esperado para este tipo de serviço. O modelo de tratamento oferecido tem características ambulatorial e médico-psiquiátrica. torno de humor e 17,0\% de neuróticos, nas modalidades de ambulatório médico-psiquiátrico (81,0\%), individual (23,0\%), grupal (13,0\%) e reabilitação psicossocial (4,3\%). Os CAPS de Santos atendem todas as categorias de transtornos mentais, sendo os mais freqüentes aqueles considerados mais graves, o que é esperado para este tipo de serviço. O modelo de tratamento oferecido tem características ambulatorial e médico-psiquiátrica.

Transtornos Mentais; Serviço de Saúde; Saúde Mental; Política de Saúde 


\section{Colaboradores}

S. B. Andreoli, C. C. P. B. Magalhães, D. Martin e R. M. F. Pinto colaboraram na concepção, desenho, análise e interpretação de dados. C. R. M. Bezerra, S. S. B. Ronchetti e A. L. P. Miranda participaram da concepção e coleta de dados. Todos os autores contribuíram com participação intelectual na confecção do artigo e aprovação final.

\section{Agradecimentos}

À Secretaria Municipal de Saúde de Santos, na pessoa do secretário Dr. T Söderberg, do coordenador de saúde mental SC Gaspar e dos diretores dos CAPS, pelo apoio e acesso aos serviços. À assessoria de saúde mental da DIR XX, na pessoa de PCB Calipo. À EB Carvalho, MNC Golegã, RC Leonhardt, ER Lima, LH Madeira, P Magila, FABO Micheletti, SNC Rocha, AM Silva, AB Siqueira, CR Teline. Ao Núcleo de Estatística e Metodologia Aplicadas da UNIFESP, na pessoa de KNC Areco e VA Cítero.

\section{Referências}

1. Thornicroft G, Tansella M. The mental health matrix: a pragmatic guide to service improvement. $1^{\text {st }}$ Ed. Cambridge: Cambridge University Press; 1999.

2. Pitta AMF, Silva JFF, Lacman S, Kinoshita RT, Cavalcanti MT, Valentini WH. Determinantes da qualidade de serviços de saúde mental em municípios brasileiros: estudo da satisfação com os resultados das atividades desenvolvidas por pacientes, familiares e trabalhadores dos serviços. J Bras Psiquiatr 1995; 44:441-52.

3. Fernandes AMP. Qualidade de vida de clientes de serviços de saúde mental. Rev Psiquiatr Clin 1999; 26:68-77.

4. World Health Organization. The world health report 2001. Mental health: new understanding new hope. Geneva: World Health Organization; 2001.

5. Kessler RC. Psychiatric epidemiology: selected recent advances and future directions. Bull World Health Organ 2000; 78:464-74.

6. Andreoli SB, Gastal FL, Leite SO, Welham J, McGrath J. Age-at-first-registration in schizophrenia: a comparison of mental health registers from Australia and Brazil. Schizophr Res 2002; 54:277-9.
7. Secretaria de Estado da Saúde de São Paulo. DIR XIX-BS. Dados sobre internação de pacientes psiquiátricos. Central de vagas hospitalares da baixada santista: Santos. Santos: Secretaria de Estado da Saúde de São Paulo; 2001.

8. Almeida-Filho N, Mari JJ, Coutinho E, Fanca JF Fernandes J, Andreoli SB, et al. Brazilian multicentric study of psychiatric morbidity: methodological feature and prevalence estimates. $\mathrm{Br} \mathrm{J}$ Psychiatry 1997; 171:524-9.

9. Gastal FL, Andreoli SB, Quintana MI, Almeida Gameiro M, Leite SO, McGrath J. Predicting the revolving door phenomenon among patients with schizophrenic, affective disorders and non-organic psychoses. Rev Saúde Pública 2000; 34:280-5.

10. Menezes PR, Mann AH. The social adjustment of patients with schizophrenia: implications to the mental health policy in Brazil. Rev Saúde Pública 1993; 27:340-9.

Apresentado em 28/Mar/2003

Versão final reapresentada em 23/Dez/2003 Aprovado em 05/Fev/2004 